\title{
Oficinas culinárias como estratégia de promoção de saúde em um serviço de residência terapêutica: uma abordagem multiprofissional em tempos de pandemia
}

\author{
Cuisine workshops as a health promotion strategy in a therapeutic residence service: a multiprofessional approach in \\ pandemic times \\ Talleres de cocina como estrategia de promoción de la salud en un servicio de residencia terapéutica: un enfoque \\ multiprofesional en tiempos de pandemia
}

\section{RESUMO}

Objetivo:Relatar, sob uma perspectiva multiprofissional, experiências de intervenções de educação nutricional, por meio de oficinas culinárias, com moradores de um Serviço de Residência Terapêutica em meio a atual pandemia causada pelo novo coronavírus. Método: Estudo descritivo com abordagem qualitativa, do tipo relato de experiência realizado por meio de oficinas culinárias. Resultados: Este tipo de ação favorece o estímulo sensoperceptivo, além da promoção da autonomia dos envolvidos no preparo do próprio alimento.Conclusão: A adesão dos participantes as ações, aponta para o fato de que esta atividade possibilitou o interesse em consumir alimentos mais saudáveis, destacando-se como ferramenta para melhoria dos hábitos alimentares, promoção da saúde e prevenção de doenças, como também para estimular a pesquisa e as práticas para a promoção da atenção integral à saúde mental, que ainda é limitada na formação em Saúde no Brasil.

DESCRITORES: Assistência em Saúde Mental; Promoção da Saúde; Pandemia.

\section{ABSTRACT}

Objective: To report, in a multiprofessional perspective, experiences of nutrition education interventions, through culinary workshops, with residents of a Therapeutic Residence Service in the midst of the current pandemic caused by the new coronavirus. Methods: Descriptive study with a qualitative approach, an experience report carried out through culinary workshops. Results: This type of action favors sensory-perceptual stimulation, in addition to promoting the autonomy of those involved in preparing their own food. Conclusion: Participants' adherence to the action points to the fact that this activity made it possible to have an interest in consuming healthier foods, standing out as a tool for improving eating habits, promoting health and preventing diseases, as well as to stimulate research and practices promoting comprehensive mental health care, which is still limited in health education in Brazil.

DESCRIPTORS: Mental Health Assistance; Health Promotion;Pandemic

\section{RESUMEN}

Objetivo: Informar, desde una perspectiva multiprofesional, experiencias de intervenciones de educación nutricional, a través de talleresculinarios, con residentes de unServicio de ResidenciaTerapéuticaenmedio de laactual pandemia provocada por elnuevocoronavirus. Método: Estudiodescriptivocon enfoque cualitativo, relato de experiencia realizado a través de talleresculinarios. Resultados: Este tipo de acción favorece laestimulación sensorial-perceptiva, además de promover laautonomía de quienesparticipanenlapreparación de supropia comida. Conclusión: La adhesión de los participantes a lasaccionesapunta a que estaactividadpermitiótenerinterésen consumir alimentos más saludables, destacándose como una herramienta para mejorarlos hábitos alimentarios, promover lasalud y prevenir enfermedades, así como estimular lainvestigación y prácticas para promover laatención integral de lasalud mental, que aún es limitada enlaeducación para lasaluden Brasil.

DESCRIPTORES: Atención a laSalud Mental; Promoción de laSalud; Pandemia.

RECEBIDO EM: 31/03/2021 APROVADO EM: 30/04/2021 


\section{artigo}

Dantas, M.B., Medeiros, M. G, Silva, C. S, Batista, J. M., Santos, R. A.,

Oficinas culinárias como estratégia de promoção de saúde em um serviço de residência terapêutica, uma abordagem multiprofissional em tempos de pandemia

\section{MAIARA BEZERRA DANTAS}

Graduada em Enfermagem pela Universidade Regional do Cariri (URCA).Residente no Programa de Residência Multiprofissional em Atenção Básica pela Escola Multicampi de Ciências Médicas (EMCM) - Universidade Federal do Rio Grande do Norte (UFRN).

ORCID: 0000-0002-0389-6792

\section{MIKAELE GARCIA DE MEDEIROS}

Graduada em Odontologia pela Universidade do Estado do Rio Grande do Norte (UERN). Residente no Programa de Residência Multiprofissional em Atenção Básica pela Escola Multicampi de Ciências Médicas (EMCM) - Universidade Federal do Rio Grande do Norte (UFRN).

ORCID: 0000-0002-5307-9782

\section{CECÍLIA DOS SANTOS SILVA}

Graduanda do curso de Bacharelado em Nutrição pela Universidade Federal de Campina Grande (UFCG). Membro do Grupo de Pesquisa e Estudos em Atualidades da Nutrição Clínica - CLINUTRI.

ORDCID: 0000-0002-4009-8159

\section{JOSEMÁRIA DE MEDEIROS BATISTA}

Graduada em Nutrição pela Universidade Federal do Rio Grande do Norte( UFRN). Especialista em Saúde Coletiva e Saúde da Família pela Faculdade Santa Maria. Mestranda em Práticas de Saúde e Educação pela Universidade Federal do Rio Grande do Norte(UFRN.

ORCID: 000-0002-5341-3496

\section{RICARDO ALEXANDRE DOS SANTOS}

Graduado em Enfermagem pela. Especialista em Preceptoria no SUS pelo Hospital Sírio-Libanês, São Paulo, Brasil. Especialista em Enfermagem em Psiquiatria e Saúde Mental do Centro de Ensino Superior Dom Alberto, Santa Cruz Do Sul, Brasil. ORCID: 0000-0003-4121-0912

\section{INTRODUÇÃO}

A Organização Mundial da Saúde (OMS) declarou em dezembro de 2019, o surgimento de uma nova doença, chamada de COVID-19, provocada por um vírus do tipo coronavírus denominado SARS-CoV-2, com o primeiro caso identificado em Wuhan, cidade chinesa com 11 milhōes de habitantes, e em março de 2020, foi classificada como uma emergência de saúde pública de interesse internacional, com alto risco de transmissão caracterizando-se como uma pandemia.

Segundo a OMS, aproximadamente $20 \%$ dos casos detectados requerem atendimento hospitalar por apresentarem dificuldade respiratória, dos quais aproximadamente $5 \%$ podem necessitar de suporte ventilatório.Após a identificação e aumento dos casos no Brasil, foi estabelecido o regime de quarentena juntamente com a adoção de medidas sanitárias para conter o avanço do vírus e não haver um colapso no sistema de saúde.

A quarentena estabeleceu um novo modelo das relaçōes de trabalho, passando a exigir uma maior dinâmica e adaptação dos profissionais e população no geral. Nesse contexto os serviços de saúde mental tiveram suas atividades interrompidas ou suspensas temporariamente, deixando uma lacuna no cuidado de pessoas com transtornos mentais, neurológicos ou de uso de substâncias psicoativas.

Dentre esses serviços de atendimento à saúde mental destacamos os Serviços deResidência Terapêutica (SRT), que representam alternativas de moradia para um grande contingente de pessoas que foram internadas há anos em hospitais psiquiátricos por não contarem com suporte adequado na comunidade.São casas localizadas no espaço urbano, constituídas para responder às necessidades de moradia de pessoas portadoras de transtornos mentais graves, institucionalizadas ou não.

Os domiciliados, então, necessitam de uma atenção e acompanhamento multidisciplinar na perspectiva de ressocialização e melhoria da qualidade de vida, através de atividades terapêuticas. $\mathrm{O}$ acompanha-
A quarentena

estabeleceu um novo modelo das relações de trabalho, passando a exigir uma maior dinâmica e adaptação dos profissionais e população no geral 
mento multiprofissional é realizado por uma equipe composta de profissionais da saúde em algumas áreas de formação, com objetivo de realizar ações interdisciplinares considerando aspectos biopsicossocial dos indivíduos.

Assim, com a alteração da rotina dos moradores da residência devido a pandemia, e com o objetivo de amenizar os efeitos do isolamento foram propostas a elaboração de oficinas culinárias através de uma equipe multiprofissional com a finalidade de estimular a ingestão de alimentos saudáveis, promover a autonomia e participação na escolha dos alimentos e estimular a comensalidade, buscando assim a promoção e prevenção de doenças crônicas não transmissíveis.

\section{OBJETIVO}

Socializar sob uma perspectiva multiprofissional, experiências de intervenções de educação nutricional com moradores de um Serviço de Residência Terapêutica no período na Pandemia da Covid-19.

\section{METODOLOGIA}

Trata-se de um estudo descritivo com abordagem qualitativa, do tipo relato de experiência, que socializa uma vivência prática de oficinas de saúde em um Serviço de Residência Terapêutica (SRT). Este serviço localiza-se em uma cidade do interior do Estado do Rio Grande do Norte. Compõe o SRT 6 moradores, 12 profissionais que trabalham em regime de plantão, sendo estes: 1 enfermeiro, 5 cuidadores, 2 cozinheiros, 2 auxiliares de serviços gerais e 2 seguranças que trabalham no turno da noite. No ano de 2021, o SRT começou a receber o programa de Residência Multiprofissional em Atenção Básica da Universidade Federal do Rio Grande do Norte (UFRN) da cidade de Caicó, constituído por profissionais do segundo ano de Residência.

As oficinas foram realizadas no mês de março de 2021, nas dependências da Residência Terapêutica, por uma odontóloga e uma enfermeira, ambas residentes em Atenção Básica junto a nutricionista do municí- pio e por uma graduanda em nutrição. As mesmas foram idealizadas com o intuito de promover práticas educativas em saúde em meio a pandemia da Covid-19.

$\mathrm{Na}$ realização das atividades todos os participantes fizeram uso de máscaras, luvas e toucas no preparo e manuseio dos alimentos. Além disso, evitou-se contato exagerado entre os proponentes e participantes, visto que os moradores compartilham do mesmo ambiente de convivência.

A primeira atividade foi a reprodução de uma receita de cookies de aveia. Todos participaram do preparo e degustação de seus resultados. A ação iniciou-se com a exposição dos ingredientes aos participantes, após isso os profissionais de nutrição orientaram sobre o preparo dos cookies. Durante este processo houve o preparo da massa, neste momento se reuniram em duplas e foram dando forma a massa com as mãos.

A segunda ação propôs uma atividade de educação em saúde nutricional, na qual foi utilizado um Bingo de Frutas. Iniciou-se com a exposição de imagens de algumas frutas, e a partir disso os participantes iam pintando figuras com frutas iguais as $\mathrm{da}$ imagem exposta em uma cartela de bingo. E, ao longo da ação era explicado a importância de cada fruta e feito a interação entre os proponentes e os participantes, realizando perguntas como: que frutas eles mais consumiam, quais as suas preferências e se reconheciam as frutas expostas.

\section{RESULTADOS}

$\mathrm{Na}$ primeira ação foi possívelobservar uma boa interação entre os participantes, assim como a satisfação durante o contato e manuseio dos ingredientes. Este tipo de ação favoreceu o estímulo sensoperceptivo, além da promoção da autonomia dos mesmos no preparo do próprio alimento.

$\mathrm{Na}$ segunda ação houve alguns momentos de dispersão de alguns participantes, onde os facilitadores sempre observavam a necessidade de chamar a atenção de volta à atividade. Entretanto, outros participaram ativamente e interagiram bem. Após a ação surgiram algumas dúvidas sobre os alimentos, as quais foram sanadas pelos profissio- nais de nutrição.

Estas ações explicitam sua importância devido a estimulação através de artifícios visuais e lúdicos, favorecendo a detenção da atenção dos participantes para as atividades e concomitantemente a promoção de saúde mental e nutricional. Observou-se também o bem-estar ao preparar seu próprio alimento e ver o resultado final, efetivando esta satisfação.

\section{DISCUSSÃO}

O serviço de Residência Terapêutica é caracterizado por casas em espaços urbanos desenvolvidas para o acolhimento de pessoas com transtornos mentais graves, institucionalizadas ou não. Essas moradias podem comportar de 1 a 8 moradores, e devem ter suporte profissional de acordo com a demanda de cada morador.

Os residentes do SRT apresentam especificidades quanto ao desenvolvimento de algumas atividades, isto se dá devido a algumas reações advindas do uso intenso e prolongado de psicotrópicos em decorrência dos transtornos mentais apresentados. $\mathrm{O}$ uso de psicotrópicos trazem algumas consequências como sono em demasia, perda de memória, tremores, assim como a dependência de seus usuários causam ainda a lentificação psicomotora, aumento de peso e prejuízos à saúde física.

Os moradores do SRT desta cidade contam com o apoio do Centro de Atenção Psicossocial-III (CAPS-III). Com a evolução da pandemia da Covid-19 para o ano de 2021, os serviços de Terapia Ocupacional e atividades antes prestados pelo CAPS-III a estes moradores foram reduzidas, deste modo, os moradores que antes iam diariamente para este serviço passaram a ir dois dias por semana, indo apenas um dos moradores.

Com esta restrição e a chegada da Residência Multiprofissional a este dispositivo de Atenção Primária em Saúde, estabeleceu-se nova rotina para estes moradores, com isso novas atividades foram iniciadas.

Logo, as oficinas culinárias propostas apresentaram-se como dispositivos para convivência, criação e reinvenção do coti- 


\section{A possibilidade desses sujeitos de se expressar, de explorar as relações \\ e de circular como cidadãos sustentam}

a inserção social.

Neste sentido, as

oficinas terapêuticas

apresentam função

psicossocial aliada

à clínica, com

possibilidade

de utilização de

diversos recursos. diano nas instituições de longa permanência, tendo em vista a necessidade de reconstrução do direito de criar, opinar, escolher e relacionar-se. A possibilidade desses sujeitos de se expressar, de explorar as relações e de circular como cidadãos sustentam a inserção social. Neste sentido, as oficinas terapêuticas apresentam função psicossocial aliada à clínica, com possibilidade de utilização de diversos recursos.

Peixoto e Silva, destacam que os indivíduos com transtornos mentais têm maior predisposição para o desenvolvimento de obesidade e sobrepeso em relação à população geral em virtude de sedentarismo, escolhas alimentares inadequadas, além de efeitos colaterais de medicações psicoativas e apontam a necessidade de um cuidado integral para este público, baseado no tratamento interdisciplinar.

Ainda segundo os autores, através de uma educação nutricional efetiva é possível melhorar os hábitos alimentares e com isso reduzir fatores risco e promover melhora da qualidade de vida, além de fomentar de forma positiva o prognóstico dos usuários. Este mesmo estudo, aponta que, por meio de fitoterápicos, alimentos funcionais como os ricos em triptofano, ômega 3, vitaminas do complexo B, zinco, é possível reduzir os efeitos colaterais de alguns fármacos utilizados no tratamento dos transtornos mentais.

As atividades de educação nutricional em um estudo realizado por Bogomolova e colaboradores (2016), comprovou mudanças comportamentais em pessoas com transtornos mentais graves, como a melhora de hábitos alimentares, independência, confiança e habilidades sociais melhoradas e mudança dos biomarcadores para fatores de risco para Doenças Cardiovasculares.

Lima (2020), traz uma importante reflexão sobre os impactos do distanciamento impostos pela Covid-19 na saúde mental em todas as faixas etárias. $\mathrm{O}$ estudo aponta a importância em se desenvolver alternativas seguras envolvendo medidas de promoção e prevenção de saúde visando mitigar os efeitos do isolamento prolongado, incluindo evitar o ócio, praticar atividades de relaxamento e atividades físicas em ambientes protegidos.
Apesar de ser um momento delicado, e muitos serviços estarem com restrições, é importante que esta população tenha momentos de lazer e tome conhecimento de medidas de prevenção em saúde e melhoria da qualidade de vida. Diante deste cenário estas atividades têm valorosa contribuição para a melhora da saúde mental, desenvolvimento de vínculos e autonomia dos mesmos.

\section{CONCLUSÃO}

A adesão dos moradores da residência terapêutica, às oficinas de culinária, aponta para o fato de que esta atividade possibilitou aos participantes o interesse em consumir alimentos mais saudáveis, que antes mesmo estando disponíveis não pareciam atrativos. Tais resultados podem ser explicados pelo fato da participação ativa dos residentes em todo processo, promover a autonomia e inclusão, além de fomentar um espaço de escuta e acolhimento. Considerando toda a especificidade do público com transtornos mentais, assim como o impacto que o isolamento e distanciamento social imposto pelo COVID-19, a prática de atividades desta natureza na rotina dos serviços de atendimento ao público de pessoas com transtornos psiquiátricos, destaca-se como ferramenta para melhoria dos hábitos alimentares, promoção da saúde $\mathrm{e}$ prevenção de doenças, como também para estimular a pesquisa e as práticas para a promoção da atenção integral à saúde mental, que ainda é limitada na formação em Saúde em nosso país. 


\section{REFERÊNCIAS}

1.Lana RM, Coelho FC, Gomes MFC, Cruz OG, Bastos LS, Villela DAM et al. Emergência do novo coronavírus (SARS-CoV-2) e o papel de uma vigilância nacional em saúde oportuna e efetiva. Cad. Saúde Pública [Internet]. 2020 [cited 2021 Mar 29]; 36(3): e00019620.Available from: http://www.scielo.br/scielo. php?script=sci_arttext\&pid=S0102-311X2020000300301\&l$\mathrm{ng}=$ en. doi.org/10.1590/0102-311x00019620.

2.Conselho Nacional de Secretarias Municipais de Saúde - CONASEMS. Guia Orientador para o enfrenta- mento da pandemia Covid-19 na Rede de Atenção à Saúde. [Internet], Brasília; 2020 [cited 2021 Mar 15].Availablefrom: https://www. conasems.org.br/orientacao_ao_gestor/guia-orientador-parao-enfrentamento-da-pandemia-covid-19-na-rede-de-atencao-a-saude/

3.World Health Organization.The impact of COVID-19 on mental, neurological and substance use services: results of a rapid assessment. [Internet] Geneva: WHO; 2020 [cited 2021 Mar 10]. Licence: CC BY-NC-SA 3.0 IGO. Available from: https://apps.who. int/iris/bitstream/handle/10665/335838/9789240012455-e ng.pdf

4.Ministério da Saúde.Residências terapêuticas: o que são, para que servem / Ministério da Saúde, Secretaria de Atenção à Saúde, Departamento de Ações Programáticas Estratégicas. - Brasília: MS; 2004 [cited 2021 Mar 20].6 p. Disponivel em: https://bvsms.saude.gov.br/bvs/publicacoes/120.pdf

5.Caldas C, Guedes M, Souza H, Oliveira I, Felisberto M, Magalhães $\mathrm{N}$ et al. A importância da equipe multidisciplinar nas oficinas terapêuticas em saúde mental [Internet]. Periodicos.faminas.edu. br. 2019 [cited 22 March 2021]. Available from: http://periodicos. faminas.edu.br/index.php/RCFaminas/article/view/434

6.Ferreira ACZ, Brusamarello T, Capistrano FC, Marin MJS, MMA. A vivência do portador de transtorno mental no uso de psicofármacos na perspectiva do pensamento complexo. Texto contexto - enferm. [Internet]. 2017 [cited 2021 Mar 30] : 26( 3 ): e1000016. Availablefrom: http://www.scielo.br/scielo. php?script=sci_arttext\&pid $=$ S0104-07072017000300306\&lng=en doi.org/10.1590/0104-07072017001000016.

7.Costa V, Souza N, Pinheiro D, Vaz L, Mecca R, Alves S.Afetos, sabores e trilhas: a oficina de culinária como operador clínico da desinstitucionalização. Revista Interinstitucional Brasileira de Terapia Ocupacional - REVISBRATO [Internet]. 2017 [citado 2021 MAR 22];1(3), 300-317. Disponivel em: https://revistas.ufrj.br/index.php/ribto/article/view/9676doi:https://doi. org/10.47222/2526-3544.rbto9676

8.Peixoto HGE, Silva LKR. Oficina de Culinária: Uma abordagem terapêutica e funcional na promoção da Saúde Mental. Com. Ciências Saúde [Internet]. 2018 [citado 2021 MAR 222]; 29(1):58-61. Disponivel em: http://www.escs.edu.br/revistaccs/ index.php/comunicacaoemcienciasdasaude/article/view/150

9.Bogomolova S, Zarnowiecki D, Wilson A, Fielder A, Procter $N$, Itsiopoulos C, O'Dea K, Strachan J, Ballestrin M, Champion A, Parletta N. Dietary intervention for people with mental illness in South Australia. Health Promot Int. [Internet] 2018 (cited 2021 Mar 22); 1;33(1):71-83. Available from: https://pubmed.ncbi.nlm. nih.gov/27476869/doi: 10.1093/heapro/daw055.

10.LIMA RC. Distanciamento e isolamento sociais pela Covid-19 no Brasil: impactos na saúde mental. Physis [Internet]. 2020 [cited 2021 Mar 25]; 30(2): e300214. Available from: http://www.scielo.br/scielo.php?script=sci_arttext\&pid=S0103-73312020000200313\&lng=en doi.org/10.1590/ s0103-73312020300214. 